\title{
Audoly-Pomeau linear law of the Gol'denveizer torus
}

\author{
Guang-Kai Song ${ }^{1}$ and Bo-Hua Sun ${ }^{1, *}$ \\ ${ }^{1}$ School of Civil Engineering \& Institute of Mechanics and Technology, \\ Xi'an University of Architecture and Technology, Xi'an 710055, China.
}

\begin{abstract}
The Gol'denveizer problem of a torus was studied analytically by Audoly and Pomeau (2002), and the accuracy of the Audoly and Pomeau linear law was confirmed numerically by Sun (2021). However, the law does not include the major radius $R$ of the torus. To find the influence of the major radius, we used finite element numerical simulation to simulate different cases, and we propose a modified Audoly and Pomeau linear law for vertical deformation, which includes $R$. A linear law of horizontal deformation is presented as well. Our studies show that the Audoly and Pomeau linear law has high accuracy. With modified vertical and horizontal deformation, a displacement-compatible relation between them is formulated.
\end{abstract}

\section{INTRODUCTION}

The toroidal shell in partial or complete form is widely used in engineering because of its high loading capacity[1]-[7]. Compared to other shells, such as spherical and cylindrical, there are many obstacles to the determination of the deformation of a torus because of its intricate topology, primarily the Gaussian curvature, $K=\frac{\sin \theta}{a(R+a \sin \theta)}$, which changes with $\theta$ :

$$
K=\frac{\sin \theta}{a(R+a \sin \theta)}= \begin{cases}<0, & \theta \in[-\pi, 0] \\ =0, & \theta=0 \\ >0, & \theta \in[0, \pi]\end{cases}
$$

Figure 1 shows the geometry of a torus.

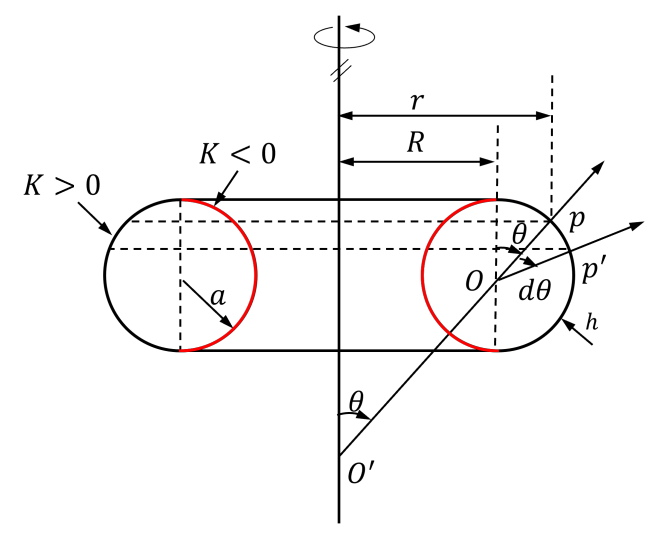

Figure 1: Geometric characteristics of torus: The radii of curvature are $R_{1}=a, R_{2}=\frac{R+a \sin \theta}{\sin \theta}$; the major curvature $K_{1}=\frac{1}{R_{1}}, K_{2}=$ $\frac{1}{R_{2}}$; so, the Gaussian curvature $K=K_{1} K_{2}=\frac{\sin \theta}{a(R+a \sin \theta)}$.

The torus has been investigated for 110 years, and many aspects have been extensively researched. In 1961, Gol'denveizer [8] identified the issue of a toroidal shell loaded under axial forces, whose outer and inner equators are loaded with opposite balanced forces, and proposed that the membrane theory of shells does not apply to this problem due to

\footnotetext{
*Corresponding author:sunbohua@xauat . edu. cn
}

the geometric features of the crown of the torus. This problem has haunted researchers for many years. Audoly and Pomeau [9][10] intensively studied the Gol'denveizer problem, and proved that Gol'denveizer was correct that the problem is not well solved by the membrane theory of shells. An approximate analytical solution of torus deformation around the crown using the membrane theory of a torus with a nonlinear boundary layer was proposed as

$$
F=\frac{4 E h^{2}}{a \sqrt{12\left(1-\mu^{2}\right)}} \Delta_{z},
$$

which is called the Audoly and Pomeau linear law, where $E$ and $\mu$ are respectively the elastic modulus and Poisson's ratio of a torus. Figure 2 is a diagram of the Gol'denveizer problem of a torus.

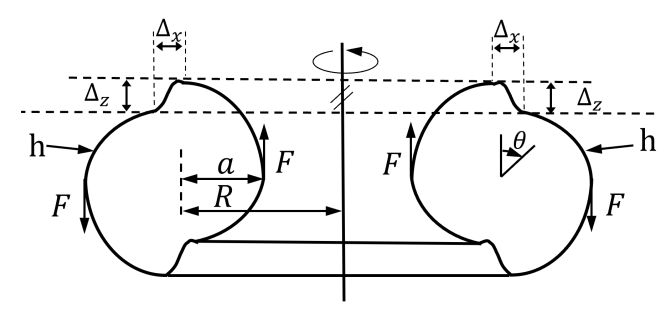

Figure 2: Gol'denveizer problem of a torus: $a$ and $R$ are the minor and major radius, respectively; $\Delta_{z}$ is the vertical displacement; $\Delta_{x}$ is the horizontal displacement; $h$ is the thickness.

We believe that eq. (2) is the first linear law for the Gol'denveizer problem, but it still has many problems [9][10]. Sun [7] researched this issue using the bending theory of shells. Sun [7] remarked that eq. (2) has high precision, and this linear law had been proved by the finite element method. However, results using the bending theory of a torus are more accurate because the linear law of Audoly and Pomeau [9] does not include the major radius of the torus. Furthermore, no one have investigated the horizontal displacement of the Gol'denveizer problem for an elastic torus. We use the finite element method to modify the linear law of Audoly and Pomeau [9], and research the horizontal displacement of the Gol'denveizer problem in the linear range. Moreover, a displacement-compatible relation between vertical and horizontal deformation is formulated. 
The remainder of this paper is organized as follows. We modify the Audoly and Pomeau linear law in Section 2. Section 3 presents the linear law of horizontal displacement of the Gol'denveizer problem, which is verified by the finite element method (FEM). The deformation compatibility equation for Gol'denveizer's problem is obtained in Section 4. We summarize our results in Section 5.

\section{MODIFICATION OF AUDOLY AND POMEAU LINEAR LAW}

To better understand the Audoly and Pomeau linear law, we used the finite element method to analyze the Gol'denveizer problem of a half-torus in the linear range. The diagram of a half-torus is presented in Figure 3. We used the ABAQUS finite element program to analyze Gol'denveizer's problem of a toroidal shell. For the numerical investigation, the Newton-Raphson solution technique (static, general) was used, with initial, minimum, and maximum step-size increments of $0.01,1 E-12$, and 0.1 , respectively. Due to the linear analysis, the Nlgeom of the static step was off. To maintain the reliability and speed of finite element calculation, reduced integration linear shell elements (S4R) were used for a half-torus. The shell edge load was considered to simulate the Gol'denveizer problem. The details of other parameters are shown in Table 1 and Figure 4.

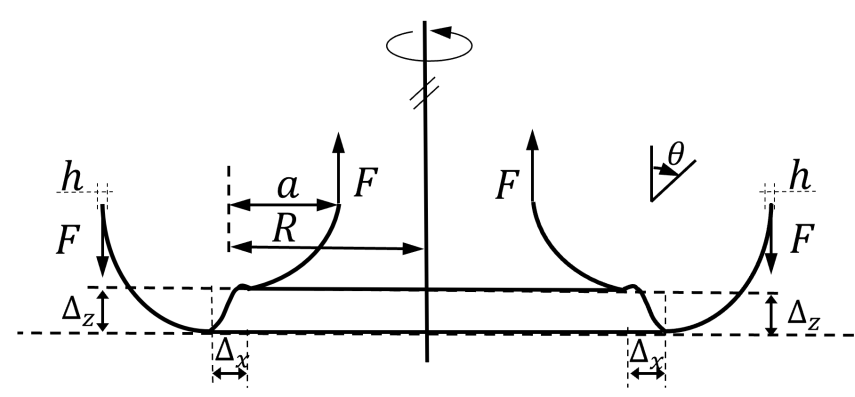

Figure 3: Geometric characteristics of half-circular torus: The shell edge load has a force of $1 N$; $a$ is the minor radius, and $R$ is the major radius; vertical displacement is $\Delta_{z}$; horizontal displacement is $\Delta_{x}$; $h$ presents the thickness.

It is worth noting that the bending theory of Sun [6] and the theory of Audoly and Pomeau [9] prove that our finite element methods can more accurately solve Gol'denveizer's problem of the torus. And this results are shown in Sun's paper[6].

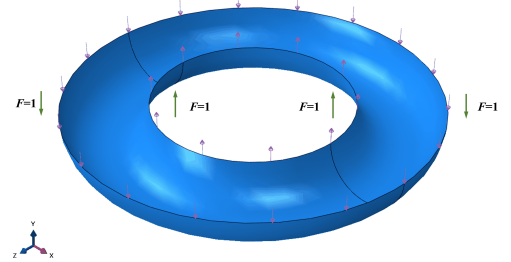

(a)Load of torus

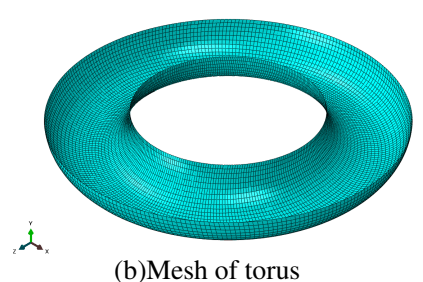

Figure 4: Details of FEM model: The load is a shell edge load, and the force is $1 N$. The mesh type is 4-node reduced integration shell elements (S4R), and the mesh size is $3 \mathrm{~mm}$.

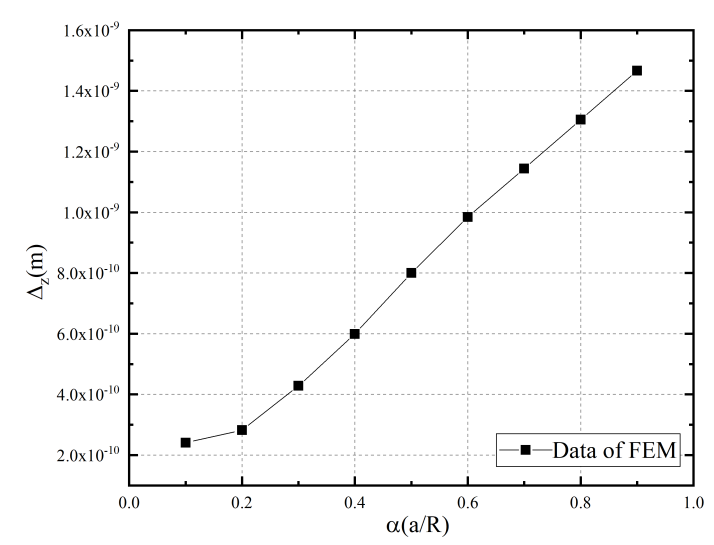

Figure 5: Vertical displacements of half-circular torus: We plot FEM data for $\Delta_{z}$ (when $\theta=\pi$ ) vs. $\alpha=a / R$ ( $a$ : minor radius; $R$ : major radius).

The model is analyzed with different values of $a$; the vertical displacements at the crown $(\theta=\pi)$ are shown in Figure 5 , from which it can be seen that the vertical displacement increases with $\alpha=a / R$ added.

As remarked above, we analyzed the vertical displacements of the Gol'denveizer problem of the torus. It is known that Audoly and Pomeau [9] combined the membrane theory of a torus with the nonlinear boundary layer and obtained a linear law,

$$
F=\frac{4 E h^{2}}{a \sqrt{12\left(1-\mu^{2}\right)}} \Delta_{z} .
$$

But this linear law does not include the major radius $R$. Therefore, to solve this problem, we studied the vertical displacements of a half-circular torus using the finite element method. We used the Audoly and Pomeau linear law to obtain the vertical displacements at the crown $(\theta=\pi)$, and compared the results with those of the finite element method. We let 
Table I: Data of half-circular torus in FEM.

\begin{tabular}{|c|c|c|c|c|c|c|}
\hline Quantity $^{1}$ & $a$ & $R$ & $h$ & $E$ & $\mu$ & $F$ \\
\hline Units & $m$ & $m$ & $m$ & $N / m^{2}$ & 1 & $\bar{N}$ \\
\hline Data & $0.3 k$ & 1 & 0.05 & $2.07 \times 10^{11}$ & 0.3 & 1 \\
\hline
\end{tabular}

Table II: Vertical displacement of finite element methods and linear law [9] for Gol'denveizer problem of half-torus $\left(\Delta_{z}[m]\right)$.

\begin{tabular}{llllllll}
\hline$a(m)$ & $h(m)$ & $R(m)$ & $a / h$ & $a / R$ & $\Delta_{z}(\mathrm{FEM})$ & $\Delta_{A P(z)}$ & $\Delta_{z} / \Delta_{A P(z)}$ \\
\hline \hline 0.1 & 0.05 & 1 & 2 & 0.1 & $2.41 e-10$ & $1.60 e-10$ & 1.510 \\
\hline 0.2 & 0.05 & 1 & 4 & 0.2 & $2.82 e-10$ & $3.19 e-10$ & 0.883 \\
\hline 0.3 & 0.05 & 1 & 6 & 0.3 & $4.28 e-10$ & $4.79 e-10$ & 0.894 \\
\hline 0.4 & 0.05 & 1 & 8 & 0.4 & $5.99 e-10$ & $6.39 e-10$ & 0.938 \\
\hline 0.5 & 0.05 & 1 & 10 & 0.5 & $8.00 e-10$ & $7.98 e-10$ & 1.002 \\
\hline 0.6 & 0.05 & 1 & 12 & 0.6 & $9.84 e-10$ & $9.58 e-10$ & 1.028 \\
\hline 0.7 & 0.05 & 1 & 14 & 0.7 & $1.44 e-9$ & $1.12 e-9$ & 1.024 \\
\hline 0.8 & 0.05 & 1 & 16 & 0.8 & $1.31 e-9$ & $1.28 e-9$ & 1.022 \\
\hline 0.9 & 0.05 & 1 & 18 & 0.9 & $1.47 e-9$ & $1.44 e-9$ & 1.021 \\
\hline \multicolumn{7}{r}{ Note: Parameters are shown in Figure 3. $h$ is torus thickness; $\Delta_{z}$ is vertical displacement using finite element method. }
\end{tabular}

$\Delta_{A P(z)}$ denote the vertical displacement using the Audoly and Pomeau linear law. The forces $F$ of FEM and Audoly and Pomeau [9] were the same. Table 2 shows the data of Audoly and Pomeau and FEM. The curves of $\Delta_{z} / \Delta_{A P(z)}$ vs. $\alpha(a / R)$ are given in this paper.

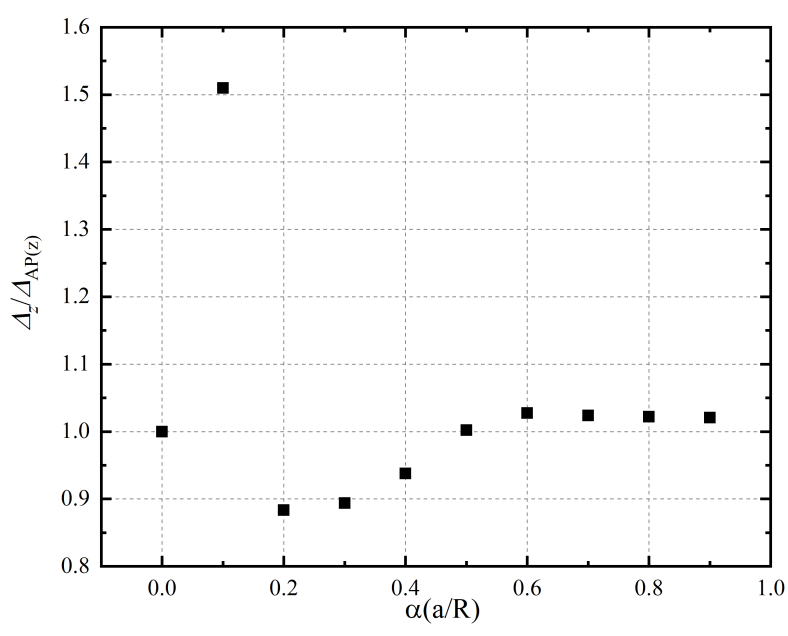

Figure 6: Plot of $\Delta_{z} / \Delta_{A P(z)}$ vs. $\alpha(a / R)$ for Gol'denveizer's problem of a half-toroidal shell. $\Delta_{z}$ and $\Delta_{A P(z)}$ are vertical displacements using finite element method and Audoly and Pomeau linear law, respectively.

In Figure $6, \Delta_{z} / \Delta_{A P(z)}$ decreases first, and then increases with $\alpha$ added. It is important to note that, when $\alpha=0.1$, the value of $\Delta_{z} / \Delta_{A P(z)}$ is 1.5 , and other values of $\Delta_{z} / \Delta_{A P(z)}$ are only in the range of $0.9 \sim 1.05$. Hence, the Audoly and Pomeau linear law [9] is inaccurate when $\alpha=0.1$. We speculate that this is caused by $a / h$. We investigated this by comparing the vertical displacement of Audoly and Pomeau and FEM for various values of $a / h$. It can be found from Table 3 that when $a / h$ is less than 2 , the Audoly and Pomeau linear law shows little agreement with the FEM results. When $a / h \leq 2$, the Audoly and Pomeau linear law [9] is no longer suitable for Gol'denveizer's problem of the elastic torus. In other words, when the torus is a thin shell, the Audoly and Pomeau linear law [9] can solve Gol'denveizer's problem with high precision.

Based on the above analysis, we need to calculate whether $a / h$ is greater than 2 when we use the Audoly and Pomeau linear law to resolve the Gol'denveizer problem. If $a / h \leq 2$, we suggest the finite element method to solve Gol'denveizer problem of torus. Furthermore, the Audoly and Pomeau linear law still faces roadblocks, such as that it does not include the major radius $R$. Therefore, we redraw the curves of $\alpha(a / R)$ vs. $\Delta_{z} / \Delta_{A P(z)}$, as shown in Figure 7.

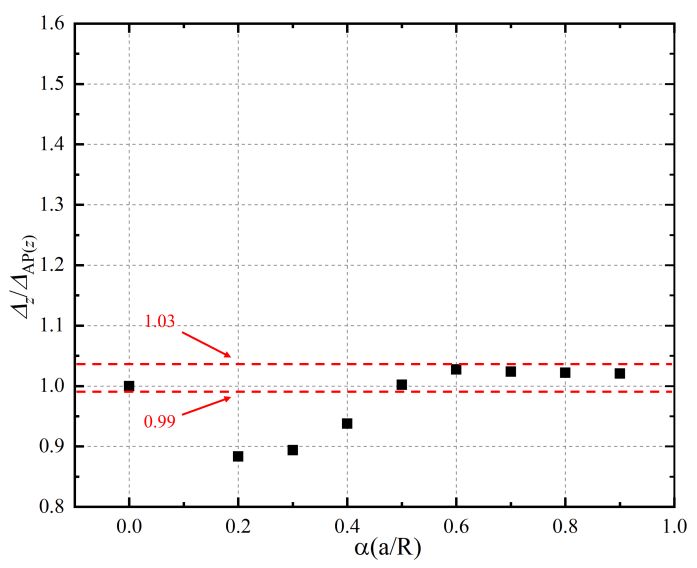

Figure 7: Scatter diagram of $\Delta_{z} / \Delta_{A P(z)}$ vs. $\alpha(a / R)$ for Gol'denveizer problem of half-toroidal shell. Not contain point that $\alpha=0.1 . \Delta_{z}$ is vertical displacement using finite element method; $\Delta_{A P(z)}$ is vertical displacement using Audoly and Pomeau linear law.

From Figure 7, when $\alpha \geq 0.5$, the value of $\Delta_{z} / \Delta_{A P(z)}$ approaches 1; hence the Audoly and Pomeau linear law has good accuracy when analyzing Gol'denveizer's problem of the elas- 
Table III: Vertical displacement for Gol'denveizer problem of a half-circular torus with various values of $a / h\left(\Delta_{z}[m]\right)$.

\begin{tabular}{llllllll}
\hline$a(m)$ & $h(m)$ & $R(m)$ & $a / h$ & $a / R$ & $\Delta_{z}(\mathrm{FEM})$ & $\Delta_{A P(z)}$ & $\Delta_{z} / \Delta_{A P(z)}$ \\
\hline \hline 0.1 & 0.05 & 1 & 2 & 0.1 & $2.41 e-10$ & $1.60 e-10$ & 1.510 \\
\hline 0.1 & 0.01 & 1 & 10 & 0.1 & $3.27 e-9$ & $3.99 e-9$ & 0.819 \\
\hline 0.07 & 0.05 & 1 & 1.4 & 0.07 & $2.87 e-10$ & $8.57 e-11$ & 3.35 \\
\hline 0.07 & 0.01 & 1 & 7 & 0.07 & $2.79 e-9$ & $2.70 e-9$ & 0.967 \\
\hline \multicolumn{7}{r}{ Note: Parameters are shown in Figure $3 . h$ represents thickness of torus; $\Delta_{z}$ is vertical displacement using finite element method. }
\end{tabular}

tic torus. But, at $\alpha<0.5$, a deviation occurs between $\Delta_{A P(z)}$ and $\Delta_{z}$. Furthermore, when $0.2 \leq \alpha<0.5, \Delta_{z} / \Delta_{A P(z)}$ decreases as $\alpha$ decreases, because when the minor and major radii have the same magnitude, the influence of the major radius $R$ on Gol'denveizer's problem of the elastic torus can be ignored. However, as $\alpha$ decreases, $a$ is smaller than $R$, major radius $R$ plays a key role in the problem. And we know that the linear law of the Audoly and Pomeau does not include the major radius $R$. So the results of Audoly and Pomeau linear law have poor consistency with those of finite element methods. Nevertheless we can deduce from the above investigation that, when $\alpha \geq 0.5$, the Audoly and Pomeau linear law is still applicable to Gol'denveizer's problem of the elastic torus.

By contrast, the Audoly and Pomeau linear law is no longer accurate when $0 \leq \alpha<0.5$. To settle this issue, we introduce $\alpha$ to this law. We produced scatter plots of $\alpha(a / R)$ vs. $\Delta_{z} / \Delta_{A P(z)}$ for the range $0 \leq \alpha<0.5$, and fitted the curve using a sine function, as shown in Figure 8 . The fitted solution

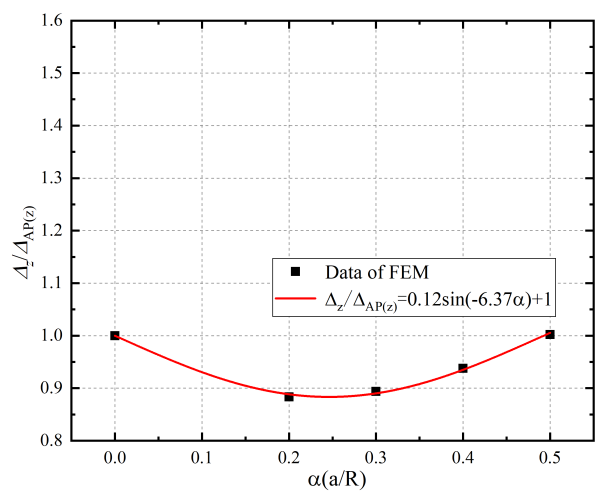

Figure 8: Modified Audoly and Pomeau linear law [9]. $\Delta_{z}$ is vertical displacement using finite element method; $\Delta_{A P(z)}$ is vertical displacement using Audoly and Pomeau linear law.

is

$$
\frac{\Delta_{z}}{\Delta_{A P(z)}}=1-0.12 \sin (6.37 \alpha) .
$$

We know that $\Delta_{A P(z)}$ is the vertical displacement using the Audoly and Pomeau linear law,

$$
F=\frac{4 E h^{2}}{a \sqrt{12\left(1-\mu^{2}\right)}} \Delta_{z}
$$

so

$$
\Delta_{A P(z)}=\frac{a \sqrt{12\left(1-\mu^{2}\right)}}{4 E h^{2}} F
$$

Substituting eq. (6) in eq. (4), we obtain

$$
\Delta_{z}=\frac{a \sqrt{12\left(1-\mu^{2}\right)}}{4 E h^{2}} F(1-0.12 \sin (6.37 \alpha)) .
$$

We know that $F=F_{s s}$, so

$$
\Delta_{z}=\frac{a \sqrt{12\left(1-\mu^{2}\right)}}{4 E h^{2}} F_{s s}(1-0.12 \sin (6.37 \alpha)) .
$$

The corrected law is

$$
F_{s s}=\frac{4 E h^{2} \Delta_{z}}{a[1-0.12 \sin (6.37 \alpha)] \sqrt{12\left(1-\mu^{2}\right)}}, \alpha=[0 \sim 0.5) \text {. }
$$

The modified Audoly and Pomeau linear law for Gol'denveizer's problem of the elastic torus $(a / h>2)$ is

$$
F_{s s}= \begin{cases}\frac{1}{1-0.12 \sin (6.37 \alpha)} \frac{4 E h^{2}}{a \sqrt{12\left(1-\mu^{2}\right)}} \Delta_{z}, & 0 \leq \alpha<0.5 \\ F, & 0.5 \leq \alpha<1,\end{cases}
$$

where $F=\frac{4 E h^{2}}{a \sqrt{12\left(1-\mu^{2}\right)}} \Delta_{z}$.

\section{HORIZONTAL DISPLACEMENT LINEAR LAW FOR GOL'DENVEIZER'S PROBLEM OF THE ELASTIC TORUS}

As above, we know that Audoly and Pomeau's [9] linear law is similar to Hooke's law. The vertical stiffness of Gol'denveizer's problem for the elastic torus is $\frac{4 E h^{2}}{a \sqrt{12\left(1-\mu^{2}\right)}}$. Therefore, we refer to eq. (5) and combine the stiffness formula of a shell structure, guessing that the linear law of horizontal displacement for Gol'denveizer's problem is

$$
F=\frac{\beta}{1-\frac{\alpha}{2}} \frac{E R}{\left[12\left(1-\mu^{2}\right)\right]^{\frac{2}{3}}} \Delta_{x},
$$

where $\beta=h / a ; a$ and $h$ are the minor radius and thickness, respectively, of the torus; $\alpha=a / R$, where $R$ is the major radius of the torus; and $E$ and $\mu$ are the elastic modulus and Poisson's ratio, respectively, of the torus.

The linear law of horizontal displacement in eq. (11) has not been validated. We verify this solution by the finite element method, using ABAQUS and the above models and parameters. The results are compared in Table 4. Our linear law of horizontal displacement of the Gol'denveizer problem of an elastic torus is in excellent agreement with FEM data. Moreover, our linear law includes the ratios $\alpha=a / R$ and $\beta=h / a$, which means that the proposed relation in eq. (11) can provide a more comprehensive study of the horizontal deformation of the Gol'denveizer problem of an elastic torus. 
Table IV: Comparison of FEM results and eq. $11\left(\Delta_{x}[m]\right)$.

\begin{tabular}{llll}
\hline Modeling & $\Delta_{x}(\alpha=0.4)$ & $\Delta_{x}(\alpha=0.5)$ & $\Delta_{x}(\alpha=0.6)$ \\
\hline \hline Linear law of horizontal displacement & $1.522 e-10$ & $1.783 e-10$ & $1.997 e-10$ \\
\hline Finite element analysis & $1.523 e-10$ & $1.697 e-10$ & $1.823 e-10$ \\
\hline
\end{tabular}

\section{DISPLACEMENT-COMPATIBLE RELATION FOR GOL'DENVEIZER'S PROBLEM OF AN ELASTIC TORUS}

We have found the linear law of horizontal and vertical displacement for Gol'denveizer's problem of the elastic torus. If we set eq. (10) equal to eq.(11), the deformation compatibility equation can be obtained.

When $0 \leq \alpha<0.5$, the deformation compatibility equation is $\frac{1}{1-0.12 \sin (6.37 \alpha)} \frac{4 E h^{2}}{a \sqrt{12\left(1-\mu^{2}\right)}} \Delta_{z}=\frac{\beta}{1-\frac{\alpha}{2}} \frac{E R}{\left[12\left(1-\mu^{2}\right)\right]^{\frac{2}{3}}} \Delta_{x}^{\text {torus. }}$

$$
\frac{\Delta_{x}}{\Delta_{z}}=\frac{1}{1-0.12 \sin (6.37 \alpha)}\left(1-\frac{\alpha}{2}\right) 4 h \frac{\left[12\left(1-\mu^{2}\right)\right]^{\frac{1}{6}}}{R}
$$

Dividing the numerator and denominator by $a$, we obtain

$$
\frac{\Delta_{x}}{\Delta_{z}}=\frac{\beta\left(4 \alpha-2 \alpha^{2}\right)\left[12\left(1-\mu^{2}\right)\right]^{\frac{1}{6}}}{1-0.12 \sin (6.37 \alpha)}
$$

When $0.5 \leq \alpha<1$,

$$
\frac{\Delta_{x}}{\Delta_{z}}=\frac{4 h\left(1-\frac{\alpha}{2}\right)\left[12\left(1-\mu^{2}\right)\right]^{\frac{1}{6}}}{R}
$$

and dividing the numerator and denominator by $a$ gives

$$
\frac{\Delta_{x}}{\Delta_{z}}=\beta\left(4 \alpha-2 \alpha^{2}\right)\left[12\left(1-\mu^{2}\right)\right]^{\frac{1}{6}}
$$

where $\beta=h / a, \alpha=a / R$.

Then the deformation compatibility equation for Gol'denveizer's problem of an elastic torus is

$$
\frac{\Delta_{x}}{\Delta_{z}}= \begin{cases}\frac{\beta\left(4 \alpha-2 \alpha^{2}\right)\left[12\left(1-\mu^{2}\right)\right]^{\frac{1}{6}}}{1-0.12 \sin (6.37 \alpha)}, & 0 \leq \alpha<0.5 \\ \beta\left(4 \alpha-2 \alpha^{2}\right)\left[12\left(1-\mu^{2}\right)\right]^{\frac{1}{6}}, & 0.5 \leq \alpha<1,\end{cases}
$$

where $\beta=h / a, \alpha=a / R$, and $\mu$ is Poisson's ratio of the

\section{CONCLUSIONS}

We used the finite element method to investigate the linearized Gol'denveizer problem for an elastic torus, and used the finite element method and the Audoly and Pomeau linear law to calculate the vertical displacement of the Gol'denveizer problem. We found that the linear law of Audoly and Pomeau [9] is no longer suitable for Gol'denveizer's problem when $a / h \leq 2$. Hence the Audoly and Pomeau linear law is applicable to the case of a torus as a thin shell. We modified this linear law, and found that when $0.5 \leq \alpha<1$, the Audoly and Pomeau linear law has good accuracy and is applicable to Gol'denveizer's problem of an elastic torus. When $0 \leq \alpha<0.5$, we modified the linear law and introduced the major radius $R$. We gave a linear law of horizontal displacement for Gol'denveizer's problem, whose accuracy was verified by the finite element method. We also gave the deformation compatibility equation for Gol'denveizer's problem of an elastic torus.
[1] Du, Qinghai, Weicheng Cui, and Bowen Zhang. Buckling characteristics of a circular toroidal shell with stiffened ribs. Ocean Engineering 108: 325-335(2015).

[2] Ross, C. T. A conceptual design of an underwater missile launcher. Ocean Engineering, 32(1), 85-99(2005).

[3] Jiammeepreecha, W, \& Chucheepsakul, S. Nonlinear static analysis of an underwater elastic semi-toroidal shell. ThinWalled Structures, 116: 12-18(2017).

[4] Clark, R. A. On the Theory of Thin Elastic Toroidal Shells. Studies in Applied Mathematics 32(1-4): 146-178(1950).

[5] V. V. Novozhilov. The theory of thin shells, Noordhoff, Groningen, (1959).

[6] Sun, B. H. Small symmetrical deformation of thin torus with circular cross-section. Thin-Walled Structures, 163, 107680(2021).

[7] Sun, B. H. Gol'denveizer's problem of elastic torus. ThinWalled Structures, 171, 108718(2022).

[8] A. L. Gol'denveizer. Theory of elastic thin shells, Pergamon Press, NewYork(1961).

[9] Audoly, B., \& Pomeau, Y. The elastic torus: Anomalous stiffness of shells with mixed type. Comptes Rendus Mécanique, 330(6): 425-432(2002).

[10] B. Audoly, Y. Pomeau. Elasticity and geometry: from hair curls to the non-linear response of shells, Oxford university press, (2010). 
Table V: Formula for Gol'denveizer's problem of a torus in this paper.

\begin{tabular}{|c|c|}
\hline$F=\frac{4 E h^{2}}{a \sqrt{12\left(1-\mu^{2}\right)}} \Delta_{z}$ & $\begin{array}{l}\text { Audoly and Pomeau linear } \\
\text { law [9] }\end{array}$ \\
\hline$F_{s s}=\left\{\begin{array}{l}\frac{1}{1-0.12 \sin (6.37 \alpha)} \frac{4 E h^{2}}{a \sqrt{12\left(1-\mu^{2}\right)}} \Delta_{z}, 0 \leq \alpha<0.5, \\
\frac{4 E h^{2}}{a \sqrt{12\left(1-\mu^{2}\right)}} \Delta_{z}, 0.5 \leq \alpha<1\end{array}\right.$ & $\begin{array}{l}\text { Modified Audoly and } \\
\text { Pomeau linear law in this } \\
\text { paper [9] }\end{array}$ \\
\hline$F=\frac{\beta}{1-\frac{\alpha}{2}} \frac{E R}{\left[12\left(1-\mu^{2}\right)\right]^{\frac{2}{3}}} \Delta_{x}$ & $\begin{array}{l}\text { Linear law of horizontal } \\
\text { displacement in this paper }\end{array}$ \\
\hline$\frac{\Delta_{x}}{\Delta_{z}}=\left\{\begin{array}{l}\frac{\beta\left(4 \alpha-2 \alpha^{2}\right)\left[12\left(1-\mu^{2}\right)\right]^{\frac{1}{6}}}{1-0.12 \sin (6.37 \alpha)}, 0 \leq \alpha<0.5, \\
\beta\left(4 \alpha-2 \alpha^{2}\right)\left[12\left(1-\mu^{2}\right)\right]^{\frac{1}{6}}, 0.5 \leq \alpha<1 .\end{array}\right.$ & $\begin{array}{l}\text { Deformation compatibility } \\
\text { equation in this paper }\end{array}$ \\
\hline
\end{tabular}

